\title{
The period map for cubic fourfolds
}

\author{
Eduard Looijenga
}

Received: 15 May 2007 / Accepted: 18 December 2008 / Published online: 31 January 2009

(C) The Author(s) 2009. This article is published with open access at Springerlink.com

\begin{abstract}
The period map for cubic fourfolds takes values in a locally symmetric variety of orthogonal type of dimension 20. We determine the image of this period map (thus confirming a conjecture of Hassett) and give at the same time a new proof of the theorem of Voisin that asserts that this period map is an open embedding. An algebraic version of our main result is an identification of the algebra of $\operatorname{SL}(6, \mathbb{C})$-invariant polynomials on the representation space $\operatorname{Sym}^{3}\left(\mathbb{C}^{6}\right)^{*}$ with a certain algebra of meromorphic automorphic forms on a symmetric domain of orthogonal type of dimension 20 . We also describe the stratification of the moduli space of semistable cubic fourfolds in terms of a Vinberg-Dynkin diagram.
\end{abstract}

Mathematics Subject Classification (2000) Primary 32G20 $\cdot$ 14J35 · Secondary 32N15

\section{Introduction}

The primitive cohomology of nonsingular cubic fourfold $Y \subset \mathbb{P}^{5}$ is located in the middle dimension (four) and has as nonzero Hodge numbers $h^{3,1}=h^{1,3}=1$ and $h_{o}^{2,2}=20$. If we make a Tate twist (which subtracts $(1,1)$ from the bidegrees), then this looks very much like the primitive cohomology of a polarized K3 surface, the difference only being that the $(1,1)$ summand is of dimension 20 instead of 19. This observation has in a sense been explained by Arnaud Beauville and Ron Donagi [2]: they showed that the Fano variety of lines on $Y$ is a deformation of the symmetric square with resolved diagonal of a polarized K3 surface (this Fano variety is an example of a complex symplectic fourfold). It was the point of departure for Claire Voisin [13] for her proof of the injectivity of the period map for cubic fourfolds (which amounts to the assertion that the polarized Hodge structure on the primitive cohomology of a nonsingular cubic fourfold determines the fourfold up to projective transformation).

The question that remained was the image of this period map. If we make the passage from the cubic fourfold to its Fano variety, then a theorem of Huybrechts [5] asserts that

E. Looijenga ( $\otimes)$

Mathematisch Instituut, Universiteit Utrecht, P.O. Box 80.010, 3508 TA, Utrecht, Netherlands

e-mail: E.J.N.Looijenga@uu.nl 
we have essentially surjectivity: every Hodge structure of this type can be realized by a deformation of a Fano variety of a cubic fourfold. However, it is not at all clear that any global deformation of a Fano variety of a cubic fourfold comes from a deformation of the fourfold. Indeed, it was suspected by Brendan Hassett that this is not the case and in a letter to the author (dated March 22, 2002) he conjectured that the image of the period map for cubic fourfolds (with innocent singularities allowed) would miss just (what we call) an arithmetic arrangement. The missing Hodge structures would be 'swallowed' by the secant variety of the Veronese surface in $\mathbb{P}^{5}$ in the sense that they only appear as the limiting Hodge structures of all possible smoothings of this variety.

The main goal of this paper is to prove Hassett's conjecture. But our proof yields more, such as a new proof of Voisin's injectivity theorem. We show that Vinberg's Dynkin diagram of an arithmetic reflection group of hyperbolic type of rank 20 gives an insightful picture of the boundary strata and their incidence relations. (Its use is analogous to a similar diagram of a rank 19 arithmetic reflection group that we obtained long ago for K3 surfaces of degree 2 and that is described in [11], p. 82ff.)

The proof employs in a fundamental way the techniques and results that we developed in [9] and (jointly with Swierstra) in [10] with applications as the present one in mind. These pertain to compactifications of varieties of the form 'locally symmetric variety minus a locally symmetric hypersurface' and associated algebra's of meromorphic automorphic forms. They are powerful enough to enable us to identify certain GIT-compactifications without detailed knowledge of that compactification and their geometry. We initially used as our GIT-input the (as yet unpublished) work by Mutsumi Yokoyama [14], but recently a more detailed classification, due to Radu Laza [6], has become available and this allowed us to shorten some of our arguments. Laza has recently used his GIT analysis to give an alternate proof of Hassett's conjecture along the lines of Shah's approach to K3 surfaces of degree two [7].

Let us now briefly comment on the contents of the individual sections. Section 1 is mostly a study of a lattice abstractly isomorphic to the primitive cohomology of a cubic fourfold. We do this via a closely related hyperbolic lattice of rank 20 which has been considered earlier by Vinberg. Among other things this leads to a classification of primitive isotropic sublattices of the primitive cohomology of a cubic fourfold.

We use these results to describe in Sect. 2 a certain locally symmetric variety of orthogonal type of dimension 20, a locally symmetric hypersurface in this variety, and a compactification of its complement (which we later identify with the moduli space of semistable cubic fourfolds).

In Sect. 3 we define the period map and state our principal result.

Section 4 reviews our (rather elementary) theory of boundary pairs in a manner that is adapted to the present situation.

Section 5 consists of computing the degree four homology of the smooth part of the two most singular semistable cubic fourfolds: the secant variety of the Veronese surface and the one defined by $Z_{0} Z_{1} Z_{2}=Z_{3} Z_{4} Z_{5}$ (known in the classical literature as the Perazzo primal).

In Sect. 6 we prove our principal result. The proof is relatively short and could have been shorter still had we not wished to include an alternative proof of Voisin's injectivity theorem. For the latter purpose we need to study in some detail the automorphism group and the deformation theory of a cubic fourfold of the form $Z_{0} Z_{1} Z_{2}=\Phi\left(Z_{3}, Z_{4}, Z_{5}\right)$, where $\Phi$ defines a nonsingular plane cubic (this fourfold has three singular points, each of type $\left.\tilde{E}_{6}\right)$. This is done in Sect. 7.

Although this paper uses much of the techniques developed in our earlier papers, we tried to make not all of these a prerequisite. Some familiarity with [9] remains indispensable however. 


\section{The primitive cohomology lattice of cubic fourfolds}

Let $\Lambda$ be an odd unimodular lattice of signature $(21,2)$ and $\eta \in \Lambda$ such that $\eta \cdot \eta=3$ and the orthogonal complement $\Lambda_{o}$ of $\eta$ is even. We denote by $\Gamma$ resp. $\hat{\Gamma}$ the stabilizer of $\eta$ resp. of $\mathbb{Z} \eta$ (or equivalently, $\Lambda_{o}$ ) in the orthogonal group of $\Lambda$. Since minus the identity is in $\hat{\Gamma}-\Gamma$, we have $\hat{\Gamma}=\{ \pm 1\} \times \Gamma$. If an element of $\hat{\Gamma}$ acts trivially on $\Lambda_{o}$, then it will leave $\eta$ fixed (for we have a natural identification of $\mathbb{Z} \eta /(3 \eta)$ with $\left.\Lambda_{o}^{*} / \Lambda_{o}\right)$, and so $\hat{\Gamma}$ acts faithfully on $\Lambda_{o}$. We may characterize $\hat{\Gamma}$ as the full orthogonal group of $\Lambda_{o}$ and $\Gamma$ as the subgroup that acts trivially on $\Lambda_{o}^{*} / \Lambda_{o}$. For reasons that become clear shortly, we will call a vector $v \in \Lambda_{o}$ a long root if $v \cdot v=2$; such a vector has the property that the orthogonal reflection in it, $s_{v}: x \mapsto x-(x \cdot v) v$, is in $\Gamma$.

We can identify $\Lambda$ with $2 E_{8} \perp 2 U \perp 3 I$ (here $E_{8}$ is the lattice spanned by a root system of that name, $U$ stands for the hyperbolic unimodular lattice of rank two and $I$ denotes the odd unimodular rank one lattice: it has a generator $\epsilon$ with $\epsilon \cdot \epsilon=1$ ) in such a manner that $\eta=\epsilon_{1}+\epsilon_{2}+\epsilon_{3}$. So then $\Lambda_{o}=2 E_{8} \perp 2 U \perp A_{2}$, where $A_{2}$ is spanned by $\beta_{1}:=\epsilon_{1}-\epsilon_{2}$ and $\beta_{2}:=\epsilon_{2}-\epsilon_{3}$. Notice that the orthogonal complement of $\beta_{2}$ in $A_{2}$ is spanned by $h_{1}:=$ $-2 \epsilon_{1}+\epsilon_{2}+\epsilon_{3}=\eta-3 \epsilon_{1}$, a vector with the property that (i) $h_{1} \cdot h_{1}=6$ and (ii) $\eta-h_{1} \in 3 \Lambda$. Indeed, the primitive hull of the span of $h_{1}$ and $\eta$ is the lattice spanned by $\epsilon_{1}$ and $\epsilon_{2}+\epsilon_{3}$, hence of type $A_{1} \perp I$ and the orthogonal complement of this lattice is $2 E_{8} \perp 2 U \perp A_{1}$. Notice that if we multiply the latter form by a minus sign, we get the primitive lattice of a K3 surface of degree two. The algebro-geometric explanation of this fact was given by Hassett in his thesis [4] (see also Remark 6.4 below).

Lemma 2.1 For a vector $h \in \Lambda_{o}$ with $h \cdot h=6$ we have $h \in \Gamma h_{1}$ resp. $h \in \hat{\Gamma} h_{1}$ if and only if $\eta-h$ is divisible by 3 resp. $\frac{1}{3} h$ generates $\Lambda_{o}^{*} / \Lambda_{o}$; in these cases we say that $h$ is special resp. that $\frac{1}{3} h$ is a short root. The orthogonal reflection in a short root preserves $\Lambda_{o}$ and hence acts as an element of $\hat{\Gamma}$.

Proof If $\eta-h$ is divisible by $3: \eta-h=3 \epsilon$, then, as we have seen in the above argument, $\eta-\epsilon$ and $\epsilon$ are perpendicular vectors of square norm 2 and 1 respectively. Since $\Lambda$ contains two summands of type $U$, there is (according to well-known result in lattice theory) a $g \in$ $\mathrm{O}(\Lambda)$ such that $g(\epsilon)=\epsilon_{1}$. Then $g(\eta-h) \in 2 E_{8} \perp 2 U \perp 2 I$ and for the same reason as above (the occurrence of $2 U$ ), there is an orthogonal transformation $g^{\prime}$ of this lattice (that we think of as an orthogonal transformation fixing $\left.\epsilon_{1}\right)$ that sends $g^{\prime}(\eta-h)$ to $\eta-h_{1}$. So $g^{\prime} g$ is an element of $\Gamma$ that sends $h$ to $h_{1}$. This implies all the assertions of the lemma, except the last. But for that we observe that $s_{h}: x \in \Lambda_{o} \mapsto x-\frac{1}{3}(x \cdot h) h$ is evidently orthogonal and preserves $\Lambda_{o}$ and hence lies in $\hat{\Gamma}$.

We denote the set of special vectors by $\mathcal{H}$. It is clear that for a short root $r$, either $3 r$ or $-3 r$ is special and that $r \cdot r=\frac{2}{3}$.

The vectors $h_{i}:=\eta-3 \epsilon_{i}(i=1,2,3)$ are all special, have zero sum and lie in the $A_{2}$ summand of $\Lambda_{o}$. Since the short and the long roots in that summand make up a root system of type $G_{2}$, we call it a $G_{2}$-summand.

Lemma 2.2 A set of special vectors spans a positive definite sublattice if and only if it is contained in a $\Gamma$-translate of $\left\{h_{1}, h_{2}, h_{3}\right\}$.

Proof Let $h, h^{\prime} \in \mathcal{H}$ be distinct and span a positive definite sublattice. Since $h, h^{\prime} \in \mathcal{H}$ span a positive definite lattice of rank two we must have $\left|h \cdot h^{\prime}\right|<6$. Since $h \cdot h^{\prime}$ is divisible by 3 , 
it must therefore lie in $\{-3,0,3\}$. We know that $h^{\prime}=h+3 v$ for some $v \in \Lambda_{o}$. From $h \cdot h^{\prime} \in$ $\{-3,0,3\}$ we get that $h \cdot v \in\{-1,-2,-3\}$. Since $h^{\prime} \cdot h^{\prime}=6$ we also have $2 h \cdot v+3 v \cdot v=0$, and so the only possibility is that $h \cdot v=-3$ and $v \cdot v=2$. This implies $h \cdot h^{\prime}=-3$. If there is a third element $h^{\prime \prime} \in \mathcal{H}-\left\{h, h^{\prime}\right\}$ such that the span of $h, h^{\prime}, h^{\prime \prime}$ is positive definite, then we have also $h^{\prime \prime} \cdot h=h^{\prime \prime} \cdot h=-3$. This implies that $h+h^{\prime}+h^{\prime \prime}$ is isotropic. As we assumed that the lattice spanned by $h, h^{\prime}, h^{\prime \prime}$ is positive definite, it follows that $h+h^{\prime}+h^{\prime \prime}=0$. So we have then a maximal subset of $\mathcal{H}$ that spans a positive definite sublattice. Returning to the pair $h, h^{\prime} \in \mathcal{H}$, then $\epsilon^{\prime}:=\frac{1}{3}\left(\eta-h^{\prime}\right), \epsilon^{\prime \prime}:=\frac{1}{3}\left(\eta-h^{\prime \prime}\right)$ and $\epsilon^{\prime \prime \prime}:=-\epsilon-\epsilon^{\prime}+\eta$ span mutually perpendicular vectors of norm 1 . We thus get an embedding $j: 3 I \rightarrow \Lambda$ that sends the sum of the generators to $\eta$. The orthogonal complement of $j$ is even, unimodular and of signature $(18,2)$, hence isomorphic to $2 E_{8} \perp 2 U$. Any vector in $3 I$ of selfproduct 3 is a signed sum of basis vectors and hence equivalent to $\eta$. This implies that $j$ may be composed with an element of $\Gamma$ to produce the given embedding of $3 I$ in $\Lambda$. This will take $h^{\prime}$ and $h^{\prime \prime}$ to $\left\{h_{1}, h_{2}, h_{3}\right\}$.

\section{Lemma 2.3 The primitive isotropic elements of $\Lambda_{o}$ lie in a single $\Gamma$-orbit.}

Proof This is again a formal consequence of the fact that $\Lambda_{o}$ contains a sublattice isomorphic to $2 U$.

The associated hyperbolic lattice An example of a primitive isotropic vector is the basis vector $e_{2}$ in the second hyperbolic summand. It is clear that $e_{2}^{\perp} / \mathbb{Z} e_{2}=2 E_{8} \perp A_{2} \perp U$. This lattice, that we shall denote by $\Lambda_{1}$, has hyperbolic signature. Let $W$ denote the group generated by reflections in all the roots (long and short) in $\Lambda_{1}^{*}$. Vinberg implemented in [12] his algorithm for finding a fundamental polyhedron of $W$ and found it terminates. He thus ended up with a finite collection $B$ of roots such that the inner product between any pair of distinct element is $\leq 0$ and that every root in $\Lambda_{1}^{*}$ is a linear combination of elements of $B$ with all coefficients in $\mathbb{Z}_{\geq 0}$ or all in $\mathbb{Z}_{\leq 0}$. This is equivalent to the Dynkin diagram $D(B)$ having the property that the vertices of every maximal subdiagram of finite resp. of pure affine type (meaning that all connected components of its are of that type) span a sublattice of corank one in $\Lambda_{1}^{*}$.

The full subdiagram $D\left(B_{\ell}\right)$ on the set of long roots $B_{\ell}$ in $B$ is given in Fig. 1. This picture reveals a symmetry that was not apparent before: we can describe the abstract graph $D\left(B_{\ell}\right)$ solely in terms of a 6-element set $\mathcal{B}$ of which is given a partition into 3-element subsets $\mathcal{B}^{\prime}, \mathcal{B}^{\prime \prime}$ ( $\mathcal{B}$ is the set of branch points of $B_{\ell}$ partitioned by the equivalence relation of being not connected by an edge): consider their join $\mathcal{B}^{\prime} \star \mathcal{B}^{\prime \prime}$ (a graph whose set of vertices is $\mathcal{B}$ and whose set of edges is the set of unordered pairs, one item in $\mathcal{B}^{\prime}$, another in $\left.\mathcal{B}^{\prime \prime}\right)$. Then $D\left(B_{\ell}\right)$ is obtained by putting on each edge of this join two additional vertices. So any new vertex has as its label an element of $\left(\mathcal{B}^{\prime} \times \mathcal{B}^{\prime \prime}\right) \cup\left(\mathcal{B}^{\prime \prime} \times \mathcal{B}^{\prime}\right)$. This is illustrated by Fig. 1, where we have denoted the elements of the two sets by $\{a, b, c\}$ and $\{u, v, w\}$. So the vertices of degree 3 of $B_{\ell}$ may be denoted $r_{a}, \ldots, r_{w}$ and those of degree 2 by $r_{a u}, \ldots, r_{w c}$. It is clear that the automorphism group of $B_{\ell}$ can be identified with the group of permutations of $\mathcal{B}$ that preserve the decomposition (which is a semidirect product $\mathbb{Z} / 2 \ltimes\left(\operatorname{Aut}\left(\mathcal{B}^{\prime}\right) \times \operatorname{Aut}\left(\mathcal{B}^{\prime \prime}\right)\right)$ ).

In $B_{\ell}$ we recognize the following maximal subdiagrams of pure affine type:

$3 \hat{E}_{6}$ : omit $\mathcal{B}^{\prime}$ or $\mathcal{B}^{\prime \prime}$,

$\hat{D}_{7} \perp \hat{A}_{11}$ : omit two degree two vertices at distance 5,

$A_{17}$ : omit the interior vertices on three disjoint lines of the join, 
Fig. 1 The long roots diagram attached to $2 E_{8} \perp A_{2} \perp U$

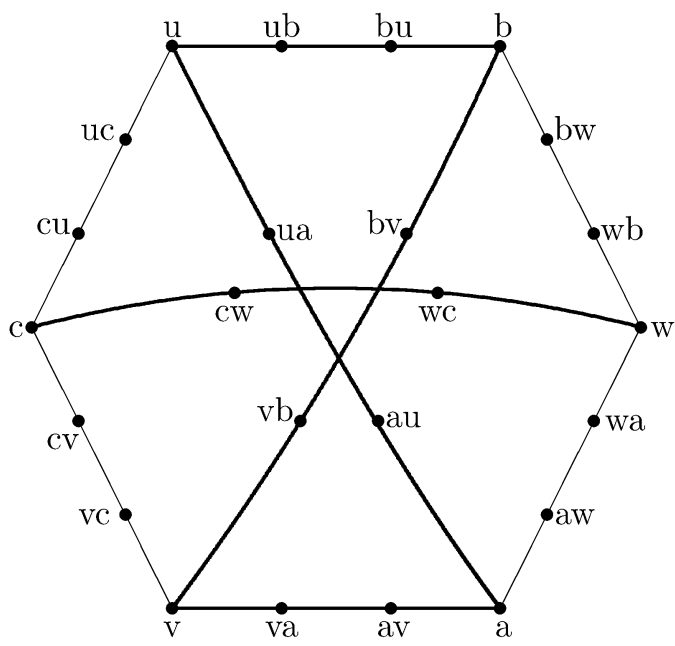

$\hat{E}_{7} \perp \hat{D}_{10}$ : omit for instance $\{a u, b v, b w, c v, c w\}$,

$\hat{D}_{16}$ : omit for instance the strings $\{a, a u, a u\},\{b v, v b\},\{c w, w c\}$,

$2 \hat{E}_{8}$ : omit for instance the string $\{a, a u, u a, u\}$ and the vertices $\{b v, c w\}$.

Only in the first two cases the corresponding subset of $B_{\ell}$ spans a sublattice of corank one. For this reason we need some short roots to produce $B$. These will then produce an extra affine summand of type $\hat{A}_{1}^{s}$ (consisting of short roots, this is what the superscript $s$ stands for) or $\hat{G}_{2}$ so that now the corank one property is fulfilled in all cases. The short roots $B_{s}$ in $B$ that we find are indexed the set of bijections from one part of $\mathcal{B}$ onto the other (so from $\mathcal{B}^{\prime}$ onto $\mathcal{B}^{\prime \prime}$ or vice versa): for such a bijection $\sigma$ the corresponding short root $r_{\sigma}$ has the following properties: $r_{\sigma}$ has inner product zero with any root of $B_{\ell}$ unless it is a degree two vertex of the form $r_{b \sigma(b)}$ for which $b$ is in the domain of $\sigma$. Furthermore, the inner product between two short roots is as follows:

$$
r_{\sigma} \cdot r_{\tau}= \begin{cases}-\frac{2}{3} & \text { if } \tau=\sigma^{-1} \text { or } \sigma \tau^{-1} \text { exists and has order 2, } \\ -\frac{4}{3} & \text { if } \sigma \tau \text { exists and has order } 2 \text { or if } \sigma \tau^{-1} \text { exists and has order 3, } \\ -\frac{5}{3} & \text { if } \sigma \tau \text { exists and has order } 3\end{cases}
$$

In the first case, $r_{\sigma}-r_{\tau}$ is isotropic and $\{\sigma, \tau\}$ defines a $\hat{A}_{1}$-subdiagram consisting of short roots (that extends in $B$ in several ways to a $\hat{G}_{2}$-diagram). In the other two cases, $r_{\sigma}, r_{\tau}$ generate a sublattice of hyperbolic signature so that $\{\sigma, \tau\}$ is of hyperbolic type.

Properties 2.4 Vinberg's theory of reflection groups in Lobatchevski disks asserts in the present case the following.

(1) The open polyhedral cone $C \subset \Lambda_{1} \otimes \mathbb{R}$ defined by $x \cdot b<0$ for all $b \in B$ is contained in a connected component $\left(\Lambda_{1} \otimes \mathbb{R}\right)_{+}$of the set $x \in \Lambda_{1} \otimes \mathbb{R}$ with $x \cdot x<0$.

(2) $C$ is a connected component in the set of $x \in \Lambda_{1} \otimes \mathbb{R}$ that lie on no reflection hyperplane.

(3) $W \bar{C}$ is the convex hull of $\overline{\left(\Lambda_{1} \otimes \mathbb{R}\right)_{+}} \cap \Lambda_{1}$ (this is also the union of $\left(\Lambda_{1} \otimes \mathbb{R}\right)_{+}$and the rays spanned by an isotropic vector in $\Lambda_{1}$ on the boundary of $\left.\left(\Lambda_{1} \otimes \mathbb{R}\right)_{+}\right)$and $\bar{C}$ is a strict fundamental domain for the action of $W$ in $\overline{\left(\Lambda_{1} \otimes \mathbb{R}\right)_{+}}$. Moreover, $W$ is generated by the reflections in the elements of $B$. 
(4) The index two subgroup of the orthogonal group of $\Lambda_{1}$ that preserves $\left(\Lambda_{1} \otimes \mathbb{R}\right)_{+}$ contains $W$ as a normal subgroup with quotient the symmetry group $\operatorname{Aut}(D(B))=$ $\mathbb{Z} / 2 \ltimes\left(\operatorname{Aut}\left(\mathcal{B}^{\prime}\right) \times \operatorname{Aut}\left(\mathcal{B}^{\prime \prime}\right)\right)$.

(5) Let $C \subset C_{s} \subset\left(\Lambda_{1} \otimes \mathbb{R}\right)_{+}$denote the connected component of the set of $x \in\left(\Lambda_{1} \otimes \mathbb{R}\right)_{+}$ that lie on no reflection hyperplane of a short root and contains $C$. Then the $W$-stabilizer of $C_{s}$ is the subgroup $W\left(B_{\ell}\right)$ of $W$ generated by the reflections in the set of long roots in $B, B_{\ell}$.

Corollary 2.5 The orbits of the primitive isotropic vectors in $\Lambda_{1}$ under the orthogonal group of $\Lambda_{1}$ are separated by the root system in their stabilizer: they are of affine type $2 \hat{E}_{8} \perp \hat{G}_{2}$, $\hat{D}_{16} \perp \hat{G}_{2}, \hat{A}_{17} \perp \hat{A}_{1}^{s}, \hat{E}_{7} \perp \hat{D}_{10} \perp \hat{A}_{1}^{s}, 3 \hat{E}_{6}, \hat{D}_{7} \perp \hat{A}_{11}$. (Here $A_{1}^{s}$ stands for a copy of $A_{1}$ spanned by a short root.)

The $\Gamma$-orbits of the primitive isotropic lattices $K \subset \Lambda_{o}$ of rank 2 can be distinguished by the isomorphism type of the associated positive definite even lattice $K^{\perp} / K$. These, in turn, can be distinguished by the root systems composed of long roots that they contain and the types that thus appear are $2 E_{8}, D_{16}, A_{17}, E_{7} \perp D_{10}, 3 E_{6}$ and $D_{7} \perp A_{11}$.

Proof We observe that the maximal subdiagrams of $D(B)$ of pure affine type are precisely the affine completions of the root systems listed and that those of a given type lie in a single $\operatorname{Aut}(B)$-orbit. In view of the Properties 2.4 above it follows that the orbits of the orthogonal group of $\Lambda_{1}$ in the set of primitive isotropic vectors are in bijection of the root system types listed.

Now let $K$ be an isotropic plane in $\Lambda_{o}$. Since its $\Gamma$-orbit is also a $\hat{\Gamma}$-orbit it is enough to show that some element of $\hat{\Gamma}$. Choose a primitive vector in $K$. Then there exists an element of $\Gamma$ that takes that element to $e_{2}$. We may therefore assume that $e_{2} \in K$. Then $K / \mathbb{Z} e_{2}$ defines a primitive isotropic rank one lattice in $\Lambda_{1}$ and the assertion follows from the discussion above.

\section{The arithmetic arrangement}

We have a quadric $\check{\mathbb{D}}$ in $\mathbb{P}\left(\Lambda_{o} \otimes \mathbb{C}\right)$ defined by $\omega \cdot \omega=0$. The open subset $\mathbb{D}$ of $\check{\mathbb{D}}$ defined $\omega \cdot \bar{\omega}<0$ has two connected components that are interchanged by complex conjugation as well as by an element of $\Gamma$. We put $X:=\Gamma \backslash \mathbb{D}$. The basic automorphic line bundle $\mathcal{A}(1)$ on $\mathbb{D}$ is the restriction of $\mathcal{O}_{\mathbb{P}\left(\Lambda_{o} \otimes \mathbb{C}\right)}^{\text {an }}(-1)$ to $\mathbb{D}$. It is acted on by $\Gamma$ and hence descends to a line bundle over $X$, denoted $\mathcal{O}_{X}(1)$, in the sense of orbifolds. (The notational switch from -1 to 1 has to do with the fact that this bundle turns out to be ample.) A section of $\mathcal{O}_{X}(k)$ is by definition a $\Gamma$-invariant section of $\mathcal{A}(k)$. The Baily-Borel theory tells us among other things that

$$
\bigoplus_{k \geq 0} H^{0}\left(X, \mathcal{O}_{X}(k)\right)=\bigoplus_{k \geq 0} H^{0}(\mathbb{D}, \mathcal{A}(k))^{\Gamma}
$$

is a finitely generated graded algebra (of automorphic forms) whose Proj defines a normal projective completion $X \subset X^{b b}$ of the orbit space. Its boundary $X^{b b}-X$ is of dimension at most one and naturally stratified: we add a singleton resp. an irreducible curve for every $\Gamma$-orbit of primitive isotropic sublattices of rank 1 resp. 2 (with the incidence relations faithfully reflecting the inclusion relations). So in the present case we have by Lemma 2.3 and Corollary 2.5 the following strata: a singleton $X(I I I)$ and irreducible curves $X(R)$, with $R$ 
running over the root systems $2 E_{8}, D_{16}, A_{17}, E_{7} \perp D_{10}, 3 E_{6}, D_{7} \perp A_{11}$. The curves have the singleton $X(I I I)$ as common boundary.

We also use the set $\mathcal{H}$ of special vectors to index the collection of hyperplanes in $\Lambda_{o} \otimes$ $\mathbb{C}$ or $\mathbb{P}\left(\Lambda_{o} \otimes \mathbb{C}\right)$ that are orthogonal to such vectors; in particular we denote by $\mathbb{D}_{h}$ the hyperplane section of $\mathbb{D}$ defined by $h \in \mathcal{H}$. Thus we get a $\Gamma$-invariant arithmetic arrangement on $\mathbb{D}$ in the sense of [9]. We denote the image of any $\mathbb{D}_{h}$ in $X$ by $X_{\mathcal{H}}$. Since $X$ is an orbifold, $X_{\mathcal{H}}$ is a Cartier divisor in $X$ the orbifold sense. Its closure $\bar{X}_{\mathcal{H}}$ in $X^{b b}$ contains a given boundary stratum if and only if there exists a special vector perpendicular to a primitive isotropic sublattice representing that stratum. This closure is disjoint with the remaining strata. Since a special vector is a multiple of a short root, we can immediately tell when this is the case:

Lemma 3.1 The closure $\bar{X}_{\mathcal{H}}$ of $X_{\mathcal{H}}$ contains the one dimensional strata of type $X(R)$ for which $R$ is a root system of rank $<18$ (so $R$ of type $2 E_{8}, D_{16}, A_{17}, E_{7} \perp D_{10}$ ) and the punctual stratum $X(I I I)$, but is disjoint with the others (the strata $X(R)$ with $R$ of type $3 E_{6}$ and $\left.D_{7} \perp A_{11}\right)$.

Notice that a subset of $\mathcal{H}$ spans a positive definite sublattice if and only if its orthogonal complement meets $\mathbb{D}$.

Corollary 3.2 to Lemma 2.2 The only proper intersections of the arrangement $\left\{\mathbb{D}_{h}\right\}_{h \in \mathcal{H}}$ are of codimension 2 and of type $G_{2}$. These lie in a single $\Gamma$-conjugacy class.

At this point we need to recall some of the results of [9], but we do that in a manner that we hope is easiest on the reader.

The closure $\overline{X_{\mathcal{H}}}$ of $X_{\mathcal{H}}$ in $X^{b b}$ is not a $\mathbb{Q}$-Cartier divisor. According to Proposition 7.2 of [9] the normalized blowup of $\overline{X_{\mathcal{H}}}$ in $X^{b b}$ that we denote here by $\widetilde{X^{b b}} \rightarrow X^{b b}$ has the property that the preimage $\widehat{X(R)} \rightarrow X(R)$ of $X(R)$ is proper and flat with fiber dimension $18-\operatorname{rk}(R)$. Such a stratum is in fact constructed in terms of a sublattice of $\Lambda_{o}$ spanned by a primitive isotropic sublattice of rank two and the special vectors perpendicular to it. The preimage $\widehat{X(I I I)} \rightarrow X(I I I)$ is of dimension two and is constructed in terms of a semipositive sublattice of $\Lambda_{o}$ of rank 3 spanned by a primitive isotropic sublattice of rank one and two special vectors perpendicular to it.

An important feature of this construction is the following: The codimension 2 intersections $\mathbb{D}_{h} \cap \mathbb{D}_{h^{\prime}}$ define in $X_{\mathcal{H}}$ a hypersurface (that we shall denote by $X_{\mathcal{H}}^{\prime}$ ) with the property that if we also blow up the strict transform of $\overline{X_{\mathcal{H}}^{\prime}}$ in $\widetilde{X^{b b}}$, then the divisors over $X_{\mathcal{H}}$ and $X_{\mathcal{H}}^{\prime}$ can be contracted in the ambient variety (in the opposite direction, like flops) onto a curve resp. a singleton. We regard this contracted variety as a compactification of $\dot{X}:=X-X_{\mathcal{H}}$. As such it is very much like the Baily-Borel compactification (which is why we shall denote it by $\dot{X}^{b b}$ ) since it may be characterized by the fact that

$$
\dot{X}^{b b}=\operatorname{Proj} \bigoplus_{k \geq 0} H^{0}(\dot{X}, \mathcal{O}(k))=\bigoplus_{k \geq 0} H^{0}(\dot{\mathbb{D}}, \mathcal{A}(k))^{\Gamma},
$$

where $\dot{\mathbb{D}}:=\mathbb{D}-\bigcup_{h \in \mathcal{H}} \mathbb{D}(h)$ (so that $\dot{X}:=\Gamma \backslash \dot{\mathbb{D}}$ ). The boundary of $\dot{X}$ in $\dot{X}^{b b}$ now comes with a decomposition into orbifolds indexed as below.

$\dot{X}\left(I_{0}\right)$ a singleton (the contraction of the divisor over $\overline{X_{\mathcal{H}}}$ ),

$\dot{X}\left(I_{1}\right)$ a curve (the contraction of the divisor over $\overline{X_{\mathcal{H}}^{\prime}}$ ), 


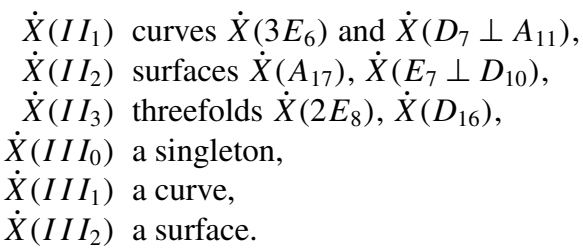

This is a stratification in the sense that the closure of a member is a union of members. The incidence scheme is dictated by lattice embeddings:

$$
\begin{array}{ccc} 
& I_{0} & < \\
& \wedge & I_{1} \\
I I I_{0} & <I I I_{1} & <I I I_{2} \\
\wedge & \hat{1} & \hat{I} \\
I I_{1} & I I_{2} & I I_{3}
\end{array}
$$

The minimal strata are the two singletons represented by $I_{0}$ and $I I I_{0}$. The maximal strata (whose closures yield the irreducible components of the boundary) are those represented at the bottom and on the right: three curves: $I_{1}=I\left(2 E_{8} \perp 2 U\right)$ and the two curves that make up $I I_{1}, I I\left(3 E_{6}\right)$ and $I I\left(D_{7} \perp A_{11}\right)$, four surfaces: $I I I_{2}=I I I\left(2 E_{8} \perp U\right)$ and the two surfaces that make up $I I_{2}: I I\left(A_{17}\right)$ and $I I\left(E_{7} \perp D_{10}\right)$, and two threefolds: $I I\left(E_{8} \perp E_{8}\right)$ and $I I\left(D_{16}\right)$ (which make up $\left.I I_{3}\right)$.

Remark 3.3 The Baily-Borel compactification $X^{b b}$ arises as the $\Gamma$-orbit space of a natural $\Gamma$-equivariant extension $\mathbb{D}^{b b} \supset \mathbb{D}$. The boundary $\mathbb{D}^{b b}-\mathbb{D}$ is naturally and $\Gamma$-invariantly decomposed into strata (in this case consisting of copies of the upper half plane and singletons) so that this stratification descends the one of the boundary $X^{b b}-X$. Something similar is the case for the compactification $\dot{X}^{b b}$ : it is obtained as the $\Gamma$-orbit space of a natural $\Gamma$ equivariant extension $\dot{\mathbb{D}}^{b b} \supset \dot{\mathbb{D}}$ whose boundary is naturally and $\Gamma$-invariantly stratified such that it descends to a stratification of $\dot{X}^{b b}-\dot{X}$.

If $S \subset \dot{\mathbb{D}}^{b b}$ is a stratum, then the group $Z_{\Gamma}(S)$ of $\gamma \in \Gamma$ that leave $S$ pointwise fixed is relevant for understanding the transversal structure of the image of $S$ in $\dot{X}^{b b}$ : the $Z_{\Gamma}(S)$-orbit space of the star of $S$ (the union of strata having $S$ in their closure) is in a natural way a normal analytic space and the natural map from that orbit space to $\dot{X}^{b b}$ is a local isomorphism along $S$. In the algebro-geometric context, the group $Z_{\Gamma}(S)$ has an interpretation as a local monodromy group. For instance, if $S$ is a singleton that lies over the singleton $\dot{X}(I I I)$, then $Z_{\Gamma}(S)$ is isomorphic the semidirect product of the Weyl group with Dynkin diagram $B_{\ell}$ (that appears in 2.4) and its root lattice $\Lambda_{1}$. The main theorem of this paper implies that this is the local monodromy group of the cubic fourfold defined by $Z_{0} Z_{1} Z_{2}=Z_{3} Z_{4} Z_{5}$ in $\mathbb{P}^{5}$.

\section{The period map}

We fix a 6-dimensional complex vector space $V$ and a generator $\mu \in \wedge^{6} V^{*}$. For some of what follows we also need a hermitian inner product on $V$ and although this serves only an auxiliary purpose, we fix that as well.

Let $Y \subset \mathbb{P}(V)$ be a cubic hypersurface (regarded as a divisor) and let $F \in \mathbb{C}[V]_{3} \cong$ $\operatorname{Sym}^{3}\left(V^{*}\right)$ be an equation for $Y$. We regard $\mu$ as a translation-invariant 6-form on $V$ so that $F^{-2} \mu$ is rational 6 -form that is invariant under scalar multiplication. The residue of this form at the hyperplane at infinity is a rational 5-form $\tilde{\omega}_{F}$ on $\mathbb{P}(V)$ with a second order pole 
along $Y$. We can take the residue once more on the smooth part $Y_{\text {reg }}$ of $Y$ in the sense of Griffiths to produce a class $\left[\omega_{F}\right] \in H^{4}\left(Y_{\text {reg }}, \mathbb{C}\right)$ : it is characterized by the fact that the value of $\left[\omega_{F}\right]$ on a 4-cycle in $Y_{\text {reg }}$ is the integral of $\tilde{\omega}_{F}$ over the pre-image of that 4-cycle in a tubular neighborhood boundary of $Y_{\text {reg }}$ in $\mathbb{P}(V)$. We can do this naturally on the form level (so that a 4-form $\omega_{F}$ on $Y_{\text {reg }}$ is defined) with the help of a hermitian inner product in $V$ (which yields a Fubini-Study metric on $\mathbb{P}(V)$ ), see [3]. This form has Hodge level 3 in the sense that it is a linear combination of a form of type $(3,1)$ and one of type $(4,0)$. It is clear that the dependence of $\omega_{F}$ on $F$ is homogeneous of degree -1 .

Suppose now that $Y \subset \mathbb{P}(V)$ is nonsingular. Then $H^{4}(Y)$ is a unimodular odd lattice of signature $(21,1)$. If $y \in H^{2}(Y)$ is the hyperplane class, then $y^{2} \in H^{4}(Y)$ has selfintersection $y^{4}=3$. The classical Lefschetz theory affirms that the orthogonal complement of $y^{2}$ in $H^{4}(Y)$ is generated by vanishing cycles. Since these have selfintersection 2 , this orthogonal complement is even. So there exists an isometry $\phi: H^{4}(Y) \rightarrow \Lambda$ that sends $y^{2}$ to $\eta$. Such an isometry is called a marking. It is clear that these markings are simply transitively permuted by $\Gamma$. It is well-known that the nonzero Hodge numbers of $Y$ in degree 4 are $h^{3,1}(Y)=$ $h^{1,3}(Y)=1$ and $h^{2,2}(Y)=21$. According to the Griffiths theory, $H^{3,1}(Y)$ is spanned by $\left[\omega_{F}\right]$ and so $\left[\omega_{F}\right] \cdot\left[\omega_{F}\right]=0$ and $\left[\omega_{F}\right] \cdot \overline{\left[\omega_{F}\right]}<0$. We also have that $[\omega] \cdot y^{2}=0$. So the marking associates to $Y$ an element of $\mathbb{D}$ (its period point). Moreover, the line in $\Lambda_{o} \otimes \mathbb{C}$ defined by that point is identified with the dual of the second tensor power of the line of equations for $Y$ (the possible $F^{\prime} s$ ). If we forget about the marking, then $Y$ defines an element of $X=\Gamma \backslash \mathbb{D}$ and the line of equations for $F$ raised to the tensor power -2 gets identified with the 'automorphic line' over that element. This identification is canonical in the sense that it is constant on the $\operatorname{GL}(V)$-orbit of $F$ in $\operatorname{Sym}^{3} V^{*}$.

We better do this universally. Let us abbreviate the GL( $V)$-representation $\operatorname{Sym}^{3} V^{*}$ by $T$ and let $\mathcal{Y} \subset \mathbb{P}(V)_{T}$ be the universal cubic. The latter is given by a single equation $F \in$ $\mathbb{C}[T \times V]$. We denote by $T^{\circ}$ the locus where $\mathcal{Y}$ is smooth over $T$ so that $\mathcal{Y}_{T^{\circ}} \subset \mathcal{Y}^{\circ}$. Denote by $\operatorname{GL}(V)$-orbit space of $T^{\circ}$ by $\mathcal{M}^{\circ}$. This is the moduli space of smooth cubic 4-folds and the previous discussion produces a morphism $P: \mathcal{M}^{\circ} \rightarrow X$ covered by an identification of $P^{*} \mathcal{A}(1)$ with $\mathcal{O}_{\mathcal{M}^{\circ}}(2)$, where $\mathcal{O}_{\mathcal{M}^{\circ}}(k)$ stands for the orbifold line bundle over $\mathcal{M}^{\circ}$ that comes from $\mathcal{O}_{\mathbb{P}(T)}(k)$.

This discussion essentially subsists if the singular points of $Y$ are all simple, that is, of type $A, D$ or $E$ : then any one parameter smoothing of $Y$ has finite monodromy so that a finite base change eliminates the monodromy altogether. By a theorem of Griffiths the period map then extends to the whole base. So if we denote the corresponding open subset of $T$ by $\dot{T}$ and denote its GL(V)-orbit space by $\dot{\mathcal{M}}$, then we have a period map

$$
P: \dot{\mathcal{M}} \rightarrow X
$$

It is well-known (and not that difficult to show) that $P$ is a local isomorphism. Much harder is the theorem of Voisin [13] which asserts that $P$ is injective. We do not want to make use that theorem, but rather wish to reprove it along the way. Our main result may be stated as follows.

Theorem 4.1 The period map for cubic fourfolds with at most simple singularities, $P$ : $\dot{\mathcal{M}} \rightarrow X$, is an open embedding with image $\dot{X}$. It identifies the automorphic line bundle restricted to $\dot{X}$ with the line bundle $\mathcal{O}_{\mathcal{M}^{\circ}}(2)$ so that we obtain an isomorphism of $\mathbb{C}$-algebras

$$
\bigoplus_{k} H^{0}(\dot{\mathbb{D}}, \mathcal{A}(k))^{\Gamma} \rightarrow \mathbb{C}\left[\operatorname{Sym}^{3} V^{*}\right]^{\operatorname{SL}(V)}
$$


which multiplies the degree by 2. The passage to Proj makes the above embedding extend to an isomorphism of the GIT completion of $\dot{\mathcal{M}}$ onto the Baily-Borel type compactification $\dot{X}^{b b}$ of $\dot{X}$.

We should perhaps point out that since $-1 \in \operatorname{SL}(V)$ acts as -1 on $\operatorname{Sym}^{3} V^{*}$, the $\operatorname{SL}(V)$ invariants on $\mathrm{Sym}^{3} V^{*}$ have even degree.

We recall that the GIT completion $\mathcal{M}$ of $\dot{\mathcal{M}}$ is $\operatorname{Proj}\left(\mathbb{C}\left[\mathrm{Sym}^{3} V\right]^{\mathrm{SL}(V)}\right.$ ) (and so implicit in this theorem is the statement that cubic fourfolds with singularities of type at most $A, D$ or $E$ are stable). The geometric invariant theory for cubic fourfolds has been worked out by Yokoyama [14] (see also Allcock [1]) and more fully by Laza [6]. We need the following.

Theorem 4.2 (Yokoyama, Laza) Every cubic fourfold with at most simple singularities is stable. Any singular point of a minimal strictly semistable cubic fourfold is simple or simple elliptic and otherwise locally analytically equivalent to the zero set $z_{1}^{2}+z_{2}^{2}+z_{3}^{2}+z_{4}^{2}\left(A_{\infty}\right)$, $z_{1}^{2} z_{2}+z_{3}^{2}+z_{4}^{2}\left(D_{\infty}\right)$ or $z_{1}^{2}+z_{2}^{3}+z_{3}^{2}$ (transversal $A_{1}$ in codimension 2 ).

The GIT boundary $\mathcal{M}-\dot{\mathcal{M}}$ is stratified with each stratum parameterizing fourfolds of the same topological type near their nonsimple singular locus. There are two minimal strata: the singleton $\dot{\mathcal{M}}\left(I_{0}\right)$ represented by the orbit of the secant variety of the Veronese variety and the singleton $\dot{\mathcal{M}}\left(I I I_{0}\right)$ representing the fourfold admitting the equation $S_{0} S_{1} S_{2}=T_{0} T_{1} T_{2}$. Only two strata are not incident with $\dot{\mathcal{M}}\left(I_{0}\right)$ : the stratum $\dot{\mathcal{M}}\left(3 E_{6}\right)$ parameterizing fourfolds with three simple singularities of type $\tilde{E}_{6}$ and another that we denote by $\dot{\mathcal{M}}\left(D_{7} \perp A_{11}\right)$. Both are of dimension one, are open in $\mathcal{M}-\dot{\mathcal{M}}$ and have $\dot{\mathcal{M}}\left(I I I_{0}\right)$ as boundary.

\section{Boundary pairs}

We shall use a technique introduced in [10] that we presently recall. If $\mathcal{Y} \subset \mathbb{P}(V) \times \Delta$ is a smoothing in $\mathbb{P}(V)$ of a cubic fourfold $Y$ (so $Y=Y_{0}$ and $Y_{t}$ is smooth for $t \neq 0$ ), then an equation for $\mathcal{Y}$ (whose coefficients are holomorphic functions on $\Delta$ ) leads via the construction in Sect. 4 to a relative 4-form $\{\omega(t)\}$ on $\left(\mathcal{Y}-Y_{\mathrm{sg}}\right) / \Delta$. Any class $u \in H_{4}\left(Y_{\text {reg }}\right)$ can be displaced to nearby fibers to produce a flat family $\left\{u(t) \in H_{4}\left(Y_{t}\right)\right\}_{t \in \Delta}$ such that $\int_{u(t)} \omega(t)$ is continuous (in fact holomorphic) on $\Delta$. Dually, we get a homomorphism $H^{4}\left(Y_{t}\right) \rightarrow H^{4}\left(Y_{\text {reg }}\right)$. The following Lemma is extracted (and a sketch of its proof reproduced) from [10].

Lemma 5.1 Let $Y \subset \mathbb{P}(V)$ be a cubic fourfold (with equation $F$ ) with the property that

$$
\int_{Y_{\mathrm{reg}}} \omega_{F} \wedge \overline{\omega_{F}}=-\infty .
$$

Let $\mathcal{Y} \subset \mathbb{P}(V) \times \Delta$ be a smoothing of $Y, S \subset \Delta^{\times}$a sector and let a marking of $\mathcal{Y}_{S} / S$ be given as an isomorphism $H^{4}\left(\mathcal{Y}_{S}\right) \cong \Lambda$ (so that is defined a period map $P: S \rightarrow \mathbb{D}$ ). If we denote by

$$
\Psi: \Lambda_{o} \subset \Lambda \cong H^{4}\left(\mathcal{Y}_{S}\right) \rightarrow H^{4}\left(Y_{\text {reg }}\right)
$$

the composite of the marking and the map defined above, then any accumulation point of $P(s), s \in S \rightarrow 0$, is contained in $\mathbb{P}\left(\operatorname{ker}\left(\psi_{\mathbb{C}}\right)\right)$.

Outline of proof Given $u \in H_{4}\left(Y_{\text {reg }}\right)$, push it to nearby fibers to produce a flat family $\{u(t) \in$ $\left.H_{4}\left(Y_{t}\right)\right\}_{t \in \Delta}$ and choose an equation for $\mathcal{Y}$ as above so that we have a relative 4 -form $\{\omega(t)\}$ 
on $\left(\mathcal{Y}-Y_{\mathrm{sg}}\right) / \Delta$ for which $\int_{u(t)} \omega(t)$ is continuous. For $t \neq 0$, we have that

$$
[\omega(t)] \cdot \overline{[\omega(t)]}=\int_{Y_{t}} \omega(t) \wedge \overline{\omega(t)} .
$$

The latter cannot have a finite limit as $t \rightarrow 0$, because $\int_{Y_{\mathrm{reg}}} \omega(t) \wedge \overline{\omega(t)}=-\infty$. This means that there exists a horizontal family $\left\{v(t) \in H_{4}\left(Y_{t}\right)\right\}_{t \in S}$ such that $\left|\int_{v(t)} \omega(t)\right| \rightarrow \infty$ as $t \rightarrow 0$ and hence that

$$
\lim _{t \rightarrow 0} \frac{\int_{u(t)} \omega(t)}{\int_{v(t)} \omega(t)}=0
$$

The assertion follows.

The assertion of this lemma is only of interest if $\psi_{\mathbb{C}} \neq 0$. Each of the following two properties (that only regard $Y$ ) implies this.

Lemma 5.2 The linear map $\psi$ is nonzero if one the following holds:

$\left(B^{\prime}\right)$ the cohomology class of $\omega_{F}$ in $H^{4}\left(Y_{\mathrm{reg}}\right)$ is nonzero or

$\left(B^{\prime \prime}\right)$ there exists a $u \in H_{4}\left(Y_{\text {reg }}\right)$ such that

$$
\left|\begin{array}{cc}
u \cdot u & \left\langle y^{2}, u\right\rangle \\
\left\langle y^{2}, u\right\rangle & y^{2} \cdot y^{2}
\end{array}\right|=3(u \cdot u)-\left\langle y^{2}, u\right\rangle^{2} \neq 0 .
$$

Proof In the first case, choose $u \in H_{4}\left(Y_{\text {reg }}\right)$ such that $\int_{u} \omega_{F} \neq 0$. An equation for $\mathcal{Y} \subset$ $\mathbb{P}(V) \times \Delta$ that specializes to $F$ determines an extension of $\omega_{F}$ to a relative form on the part where $\mathcal{Y}$ is smooth over $\Delta$. The integral of this extension over $u(t)$ is continuous on $\Delta$ and hence is nonzero near $o \in \Delta$. So the projection of $u(t), t \neq 0$, in the primitive homology of a smooth fiber is nonzero. Dually, this means that via the marking $u(t)$ projects onto an element in $\Lambda_{o} \otimes \mathbb{C}$ that is not in the kernel of $\psi_{\mathbb{C}}$.

In the second case the expression in question is the self-intersection of the primitive part of $u$. If that is nonzero, then so is the primitive part of the deformed $u$.

In the situation of Lemma 5.2 we distinguish cases as follows.

If $3(u \cdot u)-\left\langle y^{2}, u\right\rangle^{2}<0$, then $H \cap \Lambda_{o}$ has signature $(20,1)$ and hence $\mathbb{P}(H \otimes \mathbb{C})$ cannot meet the closure if $\mathbb{D}$. So this case will not occur.

(I) If $3(u \cdot u)-\left\langle y^{2}, u\right\rangle^{2}>0$, then $H \cap \Lambda_{o}$ has signature $(19,2), \mathbb{P}(H \otimes \mathbb{C})$ meets $\mathbb{D}$ in its interior and the resulting hyperplane section is two copies of the symmetric domain of the orthogonal group of $H \otimes \mathbb{R}$.

(II, III) Suppose now $3(u \cdot u)-\left\langle y^{2}, u\right\rangle^{2}=0$ and $\int_{u} \omega_{F} \neq 0$. The last condition ensures that $\tilde{u} \neq 0$ so that $H \cap \Lambda_{o}$ is of corank 1 in $\Lambda_{o}$ and the first condition then says that $H \cap \Lambda_{o}$ is degenerate with rank one dimensional nillattice. If we denote the latter by $K$, then $\mathbb{P}(H \otimes \mathbb{C})$ meets the boundary of $\mathbb{D}$ in a set that can be identified with the set of rays in $\left(\Lambda_{o} / K\right) \otimes \mathbb{R}$ on which the form is $\leq 0$ (this consists of the closures of two closed real hyperbolic disks of dimension 19).

(II) This is a subcase of the previous case and refers to the situation when there exist $u_{1}, u_{2} \in H_{4}\left(Y_{\text {reg }}\right)$ for which the intersection matrix on $y^{2}, u_{1}, u_{2}$ has rank one and for which $\int_{u_{1}} \omega_{F}$ and $\int_{u_{2}} \omega_{F}$ are $\mathbb{R}$-independent. The same argument proves that $u_{1}$ and $u_{2}$ determine a primitive isotropic lattice $K \subset \Lambda_{o}$ of rank two such that any limiting value of $P(t)$ lies in 
a codimension two linear subspace $\mathbb{P}\left(\left(K^{\perp} \cap \Lambda_{o}\right) \otimes \mathbb{C}\right)$. The latter meets the closure of $\mathbb{D}$ in the two half spheres that make up $\mathbb{P}(K \otimes \mathbb{C})-\mathbb{P}(K \otimes \mathbb{R})$. These are both one-dimensional boundary components of $\mathbb{D}$ (each in a different component of $\mathbb{D}$ ).

The following naturality property for $\psi$ is clear, but nevertheless useful.

Lemma 5.3 Suppose $Y \subset \mathbb{P}(V)$ is a cubic 4-fold and $\mathcal{Y}^{\prime} \subset \mathbb{P}(V) \times \Delta$ is a one parameter deformation of $Y$ such that every fiber satisfies $(-\infty)$. Then a smoothing of the general fiber $Y^{\prime}$ of this deformation defines a smoothing of $Y$ and if $\psi^{\prime}: \Lambda_{o} \rightarrow H^{4}\left(Y_{\mathrm{reg}}^{\prime}\right)$ is obtained after a choice of a sector and a marking over that sector, then $\psi: \Lambda_{o} \rightarrow H^{4}\left(Y_{\mathrm{reg}}\right)$ is simply the composite of $\psi^{\prime}$ with that natural map $H^{4}\left(Y_{\text {reg }}^{\prime}\right) \rightarrow H^{4}\left(Y_{\text {reg }}\right)$.

So if $\psi \neq 0$, then $\psi^{\prime} \neq 0$. In other words, if $Y$ imposes a nontrivial constraint for the period map of its smoothings, then so does $Y^{\prime}$. This is the reason why we can concentrate on the most degenerate cases.

Condition $(-\infty)$ can be verified as follows.

Lemma 5.4 Condition $(-\infty)$ is satisfied if $Y$ has a singular point which admits a localanalytic equation that is weighted homogeneous (with nonnegative weights, not all zero) such that the sum of the weights is at most twice the degree of the equation.

Proof Let $f \in \mathbb{C}\left[z_{1}, \ldots, z_{5}\right]$ a weighted homogeneous equation of such a singularity. Then $f^{-2} d z_{1} \wedge \cdots \wedge d z_{5}$ defines a relative 4-form $\omega$ on the smooth part $U$ of $f=0$. This form has a nonpositive weight, say $-d$. The form $-\omega(0) \wedge \bar{\omega}(0)$ is positive (relative to the complex orientation) wherever it is defined and $\mathbb{C}^{\times}$acts on it via the absolute value map with the nonpositive weight $-2 d$. The divergence of this integral over a neighborhood of the singular point follows easily.

\section{The boundary conditions for some semistable fourfolds}

In this section we verify the boundary conditions of Sect. 5 in a number of instances. We begin with a corollary of Lemma 5.4:

Corollary 6.1 Any minimal strictly semistable cubic threefold satisfies condition $(-\infty)$. with the variables ordered such that the weight $w_{i}$ of $z_{i}$ is zero for $i<k$ and positive for $i \geq k$

Proof According to Theorem 4.2 such a threefold has a singularity that is simple elliptic, of type $A_{\infty}, D_{\infty}$ or lies on a smooth surface of singular points with transversal type $A_{1}$. These all are weighted homogeneous with weight sum at most twice the weight of a defining equation. Now apply Lemma 5.4

We now turn our attention to the two most degenerate cases.

The secant variety of a Veronese (type $I_{0}$ ) Fix a vector space $W$ of dimension 3 and consider the space $\operatorname{Sym}^{2} W$. It has dimension 6 and parametrizes the quadratic forms on $W^{*}$. The Veronese variety is the projectivization of the cone of the elements of the pure squares in $\operatorname{Sym}^{2} W$, and so the image of $\mathbb{P}(W)$ in $\mathbb{P}\left(\operatorname{Sym}^{2} W\right)$. The secant variety $Y$ of this variety consists of the elements that can be written as the sum of two squares, in other words is the 
locus of singular quadratic expressions. Its singular part is the Veronese variety (the locus of pure squares) and hence the smooth part $Y_{\text {reg }} \subset Y$ is the locus of the quadratic expressions of exact rang 2 (both are $\mathrm{SL}(W)$-orbits).

Lemma 6.2 The natural map $H_{4}\left(Y_{\mathrm{reg}}\right) \rightarrow H^{4}(Y)$ has a kernel cyclic of order 2 and image an infinite cyclic group generated by a distinguished element $a$. A lift $u \in H_{4}\left(Y_{\mathrm{reg}}\right)$ of a satisfies $u \cdot u=3$. If $y \in H^{2}(Y)$ denotes the hyperplane class and if we identify $H^{8}(Y)$ with $\mathbb{Z}$ by means of integration over the fundamental class, then $y^{4}=3, a \cdot y^{2}=1$ and $3 a-y^{2}=2 h$ for some $h \in H^{4}(Y)$ (so that $h \cdot y^{2}=0, h^{2}=6$ and $2 h+y^{2}$ is divisible by 3 ).

Proof The map that assigns to $q \in \operatorname{Sym}^{2} W$ the minimal subspace $W_{q} \subset W$ such that $q \in$ Sym $^{2} W_{q}$ gives us a fiber bundle $Y \rightarrow \check{\mathbb{P}}(W)$ : The fiber over $\left[W^{\prime}\right] \in \check{\mathbb{P}}(W)$ is the space of nonsingular elements in $\mathbb{P}\left(\mathrm{Sym}^{2} W^{\prime}\right)$, that is the complement of the conic $C\left(W^{\prime}\right)$ that is the image of $\mathbb{P}\left(W^{\prime}\right) \rightarrow \mathbb{P}\left(\mathrm{Sym}^{2} W^{\prime}\right)$. Denote by

$$
\pi: \tilde{Y} \rightarrow \check{\mathbb{P}}(W)
$$

the projective plane bundle over whose fiber over $\left[W^{\prime}\right] \in \check{\mathbb{P}}(W)$ is $\mathbb{P}\left(\operatorname{Sym}^{2} W^{\prime}\right)$ and denote by $C \subset \tilde{Y}$ the corresponding family of conics. The evident map $\tilde{Y} \rightarrow Y$ is a resolution of singularities with $C$ as exceptional divisor. The contraction of $C$ to the Veronese variety defines a $\mathbb{P}^{1}$-bundle $C \rightarrow \check{\mathbb{P}}(W)$.

If $\zeta$ denotes the tautological plane bundle over $\check{\mathbb{P}}(W)$, then $C \rightarrow \check{\mathbb{P}}(W)$ resp. $\tilde{Y}$ is the projectivization of the vector bundle $\zeta$ resp. $\operatorname{Sym}^{2} \zeta$ over $\check{\mathbb{P}}(W)$. Denote by $u \in H^{2}(\check{\mathbb{P}}(W), \mathbb{Z})$ the positive generator, that is, the first Chern class of the line bundle $\mathcal{O}_{\check{\mathbb{P}}(W)}(1)$. We have an exact sequences

$$
\begin{gathered}
0 \rightarrow \zeta \rightarrow \mathcal{O}_{\check{\mathbb{P}}(W)} \otimes W \rightarrow \mathcal{O}_{\check{\mathbb{P}}(W)}(1) \rightarrow 0, \\
0 \rightarrow \operatorname{Sym}^{2} \zeta \rightarrow \mathcal{O}_{\check{\mathbb{P}}(W)} \otimes \operatorname{Sym}^{2} W \rightarrow \mathcal{O}_{\check{\mathbb{P}}(W)}(1) \otimes W \rightarrow 0,
\end{gathered}
$$

and hence

$$
\begin{gathered}
c(\zeta)=(1+u)^{-1}=1-u+u^{2}, \\
c\left(\operatorname{Sym}^{2} \zeta\right)=(1+u)^{-3}=1-3 u+6 u^{2} .
\end{gathered}
$$

If $x \in H^{2}(\tilde{Y})$ resp. $y \in H^{2}(\tilde{Y})$ denotes the first Chern class of $\mathcal{O}_{C}(1)$ resp. $\mathcal{O}_{\tilde{Y}}(1)$, then by a formula of Grothendieck we have that

$$
\begin{aligned}
& H^{\bullet}(C)=H^{\bullet}(\check{\mathbb{P}}(W))[x] /\left(x^{2}-u x+u^{2}\right), \\
& H^{\bullet}(\tilde{Y})=H^{\bullet}(\check{\mathbb{P}}(W))[y] /\left(y^{3}-3 u y^{2}+6 u^{2} y\right) .
\end{aligned}
$$

The restriction map $H^{\bullet}(\tilde{Y}) \rightarrow H^{\bullet}(C)$ is $H^{\bullet}(\check{\mathbb{P}}(W))$-linear, but as the restriction of $\mathcal{O}_{\tilde{Y}}(1)$ to $C$ is $\mathcal{O}_{C}(2)$, it will send $y$ to $2 x$ (notice that it is indeed true that $(2 x)^{3}-3 u(2 x)^{2}+$ $\left.6 u^{2}(2 x)=-12 u\left(x^{2}-u x+u^{2}\right)\right)$. If we feed this into the exact sequence

$$
\cdots \quad \rightarrow H^{2}(\tilde{Y}) \rightarrow H^{2}(C) \rightarrow H_{c}^{4}\left(Y_{\mathrm{reg}}\right) \rightarrow H^{4}(\tilde{Y}) \rightarrow H^{4}(C) \rightarrow \cdots
$$


then we see that the middle term is the direct sum of a copy of $\mathbb{Z} / 2$ and an infinite cyclic group generated by a class $u$ that maps to $y^{2}-2 u y+4 u^{2}$. So

$$
u^{2}=\left(y^{2}-2 u y+4 u^{2}\right)^{2}=y^{4}-4 u y^{3}+12 u^{2} y^{2}
$$

(we used that $u^{3}=0$ ). In order to compute this, we observe that $H^{8}(\tilde{Y})$ is generated by $y^{4}, u y^{3}, u^{2} y^{2}$ and that we have $u y^{3}=u\left(3 u y^{2}-6 u^{2} y\right)=3 u^{2} y^{2}$ and $y^{4}=y\left(3 u y^{2}-6 u^{2} y\right)=$ $3 u y^{3}-6 u^{2} y^{2}=3 u^{2} y^{2}$. So $u^{2} y^{2}$ is the orientation class and so

$$
a^{2}=3 u^{2} y^{2}-4.3 u^{2} y^{2}+12 u^{2} y^{2}=3 u^{2} y^{2} .
$$

So if we regard $u$ as an element of $H_{4}\left(Y_{\text {reg }}\right)$, then we see that its self-intersection is 3 . The pull back of $\mathcal{O}_{\mathbb{P}\left(\operatorname{Sym}^{2} W\right)}(1)$ along the map $\tilde{Y} \rightarrow Y \subset \mathbb{P}\left(\operatorname{Sym}^{2} W\right)$ is $\mathcal{O}_{\tilde{Y}}(1)$. So the hyperplane class of $\mathbb{P}\left(\operatorname{Sym}^{2} W\right)$ is mapped to $y$ and $a \cdot y^{2}=\left(y^{2}-2 u y+4 u^{2}\right) \cdot y^{2}=3-6+4=1$ (and from $y^{4}=3$ we see that $Y$ is indeed of degree 3 ). Now consider the projection map $\tilde{Y} \rightarrow Y$. It contracts $C$ along the projection $C \rightarrow \check{\mathbb{P}}(W)$ (a $\mathbb{P}^{1}$-bundle). This implies that we have a long exact sequence

$$
\cdots \rightarrow H^{1}(\check{\mathbb{P}}(W)) \rightarrow H^{4}(Y) \rightarrow H^{4}(\tilde{Y}) \rightarrow H^{2}(\check{\mathbb{P}}(W)) \cdots
$$

Since $H^{1}(\check{\mathbb{P}}(W))=0$ and $H^{2}(\check{\mathbb{P}}(W))$ is torsion free, the map $H^{4}(Y) \rightarrow H^{4}(\tilde{Y})$ must be a primitive embedding. Therefore, for the assertions to be proved, we may replace $Y$ by $\tilde{Y}$. It is clear that the element $h:=y^{2}-3 u y+6 y^{2}$ which evidently satisfies $2 h=3\left(y^{2}-2 u y+\right.$ $\left.4 u^{2}\right)-y^{2}$ possesses also the other desired properties.

This lemma implies that we are dealing with a type (I) situation for which the hyperplane is special:

Corollary 6.3 This $Y$ and $u$ satisfy the conditions $(-\infty)$ and $\left(B^{\prime \prime}\right)$ of Lemma's 5.1 and 5.2 with the projective hyperplane perpendicular to a special vector.

Proof A calculation near the double point locus of $Y$ shows that $\omega_{F}$ is not square integrable and we have $3(u \cdot u)-\left\langle y^{2}, u\right\rangle^{2}=3.3-1=8$, so that we are in case $I$. The smoothing and the marking determine $\tilde{u} \in \Lambda$ and $\tilde{h} \in \Lambda_{o}$ satisfying $\tilde{h} \cdot \tilde{h}=6$ and $\eta-\tilde{h}=3(\tilde{u}-\tilde{h})$. Hence $\tilde{h}$ is a special vector in $\Lambda_{o}$ and the limiting values of the period on $S$ takes values in the special hyperplane in $\mathbb{P}\left(\Lambda_{o} \otimes \mathbb{C}\right)$ perpendicular to it.

Remark 6.4 Hassett considered in [4] (especially his Theorem 4.1.1) 'linear' one parameter smoothings of this fourfold (i.e., given by an equation of the form $F+t G$ ). He shows that then the limiting mixed Hodge structure is that of K3 surface of degree two. Unfortunately we cannot implement that result here in this form, as we need this for all projective smoothings, and not just for linear ones.

The Perazzo primal (type II $I_{0}$ ) Write $V$ as a direct sum of subspaces of dimension 3 and denote its coordinates accordingly: $\left(S_{0}, S_{1}, S_{2}, T_{0}, T_{1}, T_{2}\right)$. We consider the fourfold $Y \subset \mathbb{P}^{5}$ defined by the equation $F(S, T):=S_{0} S_{1} S_{2}-T_{0} T_{1} T_{2}$. This is in fact a toric variety with the torus in question acting diagonally. The singular locus $Y_{\mathrm{sg}}$ of $Y$ is the union of lines connecting a coordinate vertex in the plane $\mathbb{P}\left(V^{\prime}\right)$ with coordinate vertex in the plane $\mathbb{P}\left(V^{\prime \prime}\right)$. The evident projection of $Y$ onto $\mathbb{P}\left(V^{\prime}\right) \times \mathbb{P}\left(V^{\prime \prime}\right)$, is a morphism away from the union coordinate 
lines in the two planes $\mathbb{P}\left(V^{\prime}\right)$ and $\mathbb{P}\left(V^{\prime \prime}\right)$. We use the standard coordinates $\left(s_{1}, s_{2}, t_{0}, t_{1}, t_{2}\right)$ on the affine open subset $U\left(S_{0}\right) \subset \mathbb{P}(V)$ defined by $S_{0} \neq 0$. So $Y \cap U\left(S_{0}\right)$ is given by putting $f(s, t)=s_{1} s_{2}-t_{0} t_{1} t_{2}$ equal to zero. Under the morphism

$$
A: \mathbb{C}^{3} \rightarrow Y, \quad(u, v, w) \mapsto(u, v w, v, w, u)
$$

the preimage of $Y_{\mathrm{sg}}$ is given by the union of the planes $u=v=0$ and $u=w=0$. So $A$

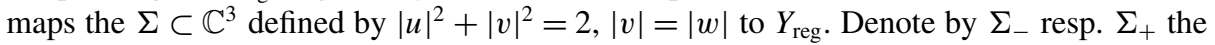
locus where $|u| \leq 1$ resp. $|u| \geq 1$. Then $(u, v, w) \in \Sigma_{-} \mapsto(u, v /|v|, w /|w|)$ identifies $\Sigma_{-}$ with a closed 2-disk times a 2-torus and $(u, v, w) \in \Sigma_{+} \mapsto(u /|u|, v, w)$ identifies $\Sigma_{+}$with a circle times the cone over a 2 -torus. This also shows that $A\left(\Sigma_{-}\right)$resp. $A\left(\Sigma_{+}\right)$is contained in $U\left(T_{0} T_{1}\right)$ resp. $U\left(S_{0} S_{1}\right)$.

The common boundary $\Sigma_{0}:=\Sigma_{-} \cap \Sigma_{+}$is a 3-torus. We orient $\Sigma_{0}$ by means of the identification with the standard 3-torus and let $\Sigma_{ \pm}$be oriented in such a manner that they induce the given orientation. Then $[\Sigma]:=\left[\Sigma_{+}\right]-\left[\Sigma_{-}\right]$is a 4-cycle. We denote by $a \in H_{4}(Y)$ the class of $A_{*}[\Sigma]$.

Lemma 6.5 We have $a \cdot a=0$ and if $y \in H^{2}(Y)$ is the hyperplane class, then $y^{2} \cdot a=0$. Moreover, the value of $\left[\omega_{F}\right] \in H^{4}\left(Y_{\mathrm{reg}}, \mathbb{C}\right)$ on a is nonzero.

Proof The locus $\Sigma^{\prime} \subset \mathbb{C}^{3}$ defined by $|u|^{2}+|v|^{2}=1,|v|=|w|$ defines a cycle [ $\left.\Sigma^{\prime}\right]$ homol-

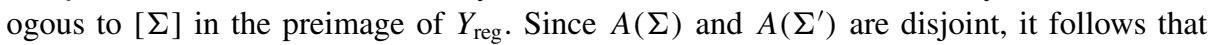
$a \cdot a=0$. Since $A$ takes values in an affine piece, we also have that $a \cdot y^{2}=0$.

In order to prove the last assertion, we identify, following Griffiths' recipe, the cohomology class on $Y_{\text {reg }}$ defined by the double residue of $F^{-2} d S_{0} \wedge \cdots \wedge d T_{2}$ as a Cech-cocycle. The corresponding rational 5-form $\tilde{\omega}_{F}$ on $\mathbb{P}(V)$ is on standard affine open piece $U\left(S_{0}\right)$ given by

$$
\tilde{\omega}_{F}=\frac{d s_{1} \wedge d s_{2} \wedge d t_{0} \wedge d t_{1} \wedge d t_{2}}{f(s, t)^{2}}
$$

We write this as the exterior derivative of a 4-form in two ways: $\tilde{\omega}_{F}=d \omega_{S_{0} S_{1}}$, where

$$
\omega_{S_{0} S_{1}}:=\left(\frac{d s_{1} \wedge d t_{0} \wedge d t_{1} \wedge d t_{2}}{s_{1} f(s, t)}\right) .
$$

This is a form defined on $U\left(S_{0} S_{1} F\right)$. We also have $\tilde{\omega}_{F}=d \omega_{T_{0} T_{1}}$, where

$$
\omega_{T_{0} T_{1}}:=\left(-\frac{d s_{1} \wedge d s_{2} \wedge d t_{0} \wedge d t_{1}}{t_{0} t_{1} f(s, t)}\right)
$$

is a form defined on $U\left(S_{0} T_{0} T_{1} F\right)$. Notice that the difference

$$
\omega_{T_{0} T_{1}}-\omega_{S_{0} S_{1}}=\frac{d f}{f} \wedge \frac{d s_{1} \wedge d t_{0} \wedge d t_{1}}{s_{1} t_{0} t_{1}}
$$

has as residue on $Y_{\text {reg }}$ the restriction $\left(s_{1} t_{0} t_{1}\right)^{-1} d s_{1} \wedge d t_{0} \wedge d t_{1}$ to $Y_{\text {reg. }}$. Let $B \rightarrow Y_{\text {reg }}$ be a tubular neighborhood of $Y_{\text {reg }}$ in $\mathbb{P}^{5}$ so that $\partial B \rightarrow Y_{\text {reg }}$ is a circle bundle with total space contained in $\mathbb{P}^{5}-Y$. An application of Stokes' theorem yields

$$
\left[\omega_{F}\right](a)=\int_{\partial B \mid A_{*}[\Sigma]} \tilde{\omega}_{F}
$$




$$
\begin{aligned}
& =\int_{\partial B \mid A_{*}\left[\Sigma_{+}\right]} d \omega_{T_{0} T_{1}}-\int_{\partial B \mid A_{*}\left[\Sigma_{-}\right]} d \omega_{S_{0} S_{1}} \\
& =\int_{\partial B \mid A_{*}\left[\Sigma_{0}\right]}\left(\omega_{T_{0} T_{1}}-\omega_{S_{0} S_{1}}\right) \quad \text { by Stokes' theorem } \\
& =\int_{\partial B \mid A_{*}\left[\Sigma_{0}\right]} \frac{d f}{f} \wedge \frac{d s_{1} \wedge d t_{0} \wedge d t_{1}}{s_{1} t_{0} t_{1}}
\end{aligned}
$$

and the residue theorem shows that the latter integral equals

$$
\int_{A_{*}\left[\Sigma_{0}\right]} \frac{d s_{1} \wedge d t_{0} \wedge d t_{1}}{s_{1} t_{0} t_{1}}=\int_{|u| 1} \frac{d u}{u} \int_{|v|=1} \frac{d v}{v} \int_{|w|=1} \frac{d w}{w}=(2 \pi \sqrt{-1})^{3} .
$$

Corollary 6.6 The Perazzo primal is a boundary case of type II or III.

\section{Proof of the main theorem}

The period map defines a morphism $P: \dot{\mathcal{M}} \rightarrow X$. It defines rational maps $G_{P}: \mathcal{M} \rightarrow X^{b b}$ and $\dot{G}_{P}: \mathcal{M} \rightarrow \dot{X}^{b b}$.

Lemma 7.1 The map $\dot{G}_{P}$ sends the boundary $\mathcal{M}-\dot{\mathcal{M}}$ to the boundary $\dot{X}^{b b}-\dot{X}$. Moreover, the preimage of $\dot{X}\left(3 E_{6}\right) \cup \dot{X}\left(D_{7} \perp A_{11}\right)$ is $\dot{\mathcal{M}}\left(3 E_{6}\right) \cup \dot{\mathcal{M}}\left(D_{7} \perp A_{11}\right)$.

Proof We established that the singleton strata $\dot{\mathcal{M}}\left(I_{0}\right)$ and $\dot{\mathcal{M}}\left(I I I_{0}\right)$ satisfy the hypotheses of Lemma's 5.1 and 5.2 with $\dot{\mathcal{M}}\left(I_{0}\right)$ of type (I) and $\dot{\mathcal{M}}\left(I I I_{0}\right)$ of type (II-III). Every stratum $S$ of $\mathcal{M}-\dot{\mathcal{M}}$ satisfies the nonsquare integrability condition $(-\infty)$ and has at least one of these singletons in its closure. Lemma 5.3 tells us that if $S \geq \dot{\mathcal{M}}\left(I I I_{0}\right)$, then $S$ is of type (II-III) and will be mapped by $G_{P}$ to the boundary $X^{b b}-X$. And if $S \geq \dot{\mathcal{M}}\left(I_{0}\right)$, then the lemma implies (in combination with Corollary 6.3) that $G_{P}$ maps $S$ to $\overline{X_{\mathcal{H}}}$. In particular, $\dot{G}_{P}$ maps $\mathcal{M}-\dot{\mathcal{M}}$ to $\dot{X}^{b b}-\dot{X}$.

Now $\dot{\mathcal{M}}\left(3 E_{6}\right)$ and $\dot{\mathcal{M}}\left(D_{7} \perp A_{11}\right)$ are the only strata of $\mathcal{M}-\dot{\mathcal{M}}$ that are not $\geq \dot{\mathcal{M}}\left(I_{0}\right)$. Likewise $X\left(3 E_{6}\right)$ and $X\left(D_{7} \perp A_{11}\right)$ are the only strata of $X^{b b}-X$ not meeting $\overline{X_{\mathcal{H}}}$. These strata are irreducible. So the preimage of the union of the latter two is the union of the former two.

The following proposition will imply Voisin's injectivity of the period map.

Proposition 7.2 (Torelli property near a boundary component) The map $G_{P}$ maps $\dot{\mathcal{M}}\left(3 E_{6}\right)$ to $\dot{X}\left(3 E_{6}\right)$ and is a local isomorphism along $\dot{\mathcal{M}}\left(3 E_{6}\right)$.

We relegate the proof to Sect. 8 .

Proof of Theorem 4.1 Lemma 7.1 implies that the $\dot{G}_{P}$-preimage of $\dot{X}\left(3 E_{6}\right)$ is $\dot{\mathcal{M}}\left(3 E_{6}\right)$ or $\dot{\mathcal{M}}\left(D_{7} \perp A_{11}\right)$ (the strata in question are irreducible). Proposition 7.2 tells us that it has to be $\dot{\mathcal{M}}\left(3 E_{6}\right)$ and that $\dot{G}_{P}$ is there a local isomorphism. This implies that $\dot{G}_{P}$ has degree one. Since $\dot{G}_{P}$ maps boundary to boundary, it follows that it restricts to a proper morphism $P: \dot{\mathcal{M}} \rightarrow \dot{X}$ of degree one. As this is a local isomorphism of degree one between integral varieties, this restriction must be an isomorphism. This isomorphism takes 
the automorphic line bundle $\mathcal{O}_{X}(1)$ to $\mathcal{O}_{\dot{\mathcal{M}}}(2)$. So it induces an isomorphism of the algebra $\bigoplus_{k} H^{0}\left(\dot{X}, \mathcal{O}_{X}(k)\right)=\bigoplus_{k} H^{0}(\dot{\mathbb{D}}, \mathcal{A}(k))^{\Gamma}$ onto the even part of $\bigoplus_{k} H^{0}\left(\dot{\mathcal{M}}, \mathcal{O}_{\dot{\mathcal{M}}}(k)\right)=$ $\mathbb{C}\left[\operatorname{Sym}^{3} V^{*}\right]^{\operatorname{SL}(V)}$ (degrees are multiplied by two). Since $-1 \in \operatorname{SL}(V)$ acts in $\operatorname{Sym}^{3} V$ as minus the identity, the odd part of the latter algebras is zero. Passing to the projs of these algebra yields that $G_{P}$ is in fact the graph of an isomorphism.

\section{Proof of the Torelli property 7.2}

We first prepare the setting. Decompose $V$ into two three dimensional subspaces $V=V^{\prime} \oplus$ $V^{\prime \prime}$ and choose coordinates $S_{0}, S_{1}, S_{2}, T_{0}, T_{1}, T_{2}$ accordingly. An element of $\dot{X}\left(3 E_{6}\right)$ is represented by a cubic fourfold $Y \subset \mathbb{P}(V)$ that has an equation of the form $F(S, T)=$ $S_{0} S_{1} S_{2}-\Phi\left(T_{0}, T_{1}, T_{2}\right)$, where $\Phi$ defines a nonsingular cubic plane curve $C \subset \mathbb{P}\left(V^{\prime \prime}\right)$. So $Y \cap \mathbb{P}\left(V^{\prime \prime}\right)=C$.

It is clear that $Y \cap \mathbb{P}\left(V^{\prime}\right)$ consists of three coordinate lines in $\mathbb{P}\left(V^{\prime}\right)$. The vertices $p_{0}, p_{1}, p_{2}$ of this coordinate triangle ( $p_{i}$ is defined by putting all coordinates but $S_{i}$ equal to nonzero) are the singular points of $Y$. The singularity at $p_{i}$ is exhibited on the affine piece defined by $S_{i}=0$; for instance, for $i=0$, we find the equation $s_{1} s_{2}=\Phi\left(t_{0}, t_{1}, t_{2}\right)$ and so it is a singular point of type $\tilde{E}_{6}$. Similarly for $p_{1}$ and $p_{2}$.

Choose a smoothing $\mathcal{Y} / \Delta$ of $Y$ and let $Y_{t}$ be a smooth fiber of it.

Lemma 8.1 The image I of $H_{4}\left(Y_{\mathrm{reg}}\right) \rightarrow H_{4}\left(Y_{t}\right)$ is a primitive isotropic sublattice. We have a canonical identification of I with $H_{1}(C)$ and the long roots in $I^{\perp} / I$ span a lattice $Q$ which decomposes into three $E_{6}$ lattices $Q_{i}, i=0,1,2$, so that the preimage of $Q_{i}$ in $I^{\perp}$ is the vanishing (Milnor) lattice of $p_{i}$. The index of $Q$ in $I^{\perp} / I$ is three.

Proof The link $L$ of $p_{0}$ in $Y$ has $H_{4}(L)$ isotropic of rank 4. We know from singularity theory that there is a canonical isomorphism $H_{4}(L) \cong H_{1}(C)$ and that the form $\omega_{F}$ maps $H_{4}(L)$ onto a lattice in $\mathbb{C}$. This last fact implies that $H_{4}(L)$ embeds in $H_{4}\left(Y_{t}\right)$. We provisionally denote the image of this embedding by $I_{0}$.

We first verify that $I_{0}$ is primitive. If $F_{t} \subset Y_{t}$ denotes the Milnor fiber of $p_{0}$ in $Y_{t}$, then it well-known that $H_{4}(L) \rightarrow H_{4}\left(F_{t}\right)$ is primitive (for $L$ can be identified with a boundary of $F$ and $H_{4}\left(F_{t}, \partial F_{t}\right) \cong H^{4}\left(F_{t}\right)$ is known to be torsion free). It therefore suffices to show that $H_{4}\left(F_{t}\right) \rightarrow H_{4}\left(Y_{t}\right)$ is primitive. But $Y_{t} / F_{t}$ is homeomorphic with the fourfold $Y^{\prime}$ that we obtain by smoothing the points $p_{1}, p_{2}$ of $Y$, while retaining $p_{0}$. So it suffices to show that $H_{4}\left(Y^{\prime}\right)$ is torsion free. This follows from the following general argument: let $X \subset Y^{\prime}$ be a generic hyperplane section (so $X$ is a smooth cubic fourfold) and consider the exact sequence

$$
H_{4}\left(Y^{\prime}-Z\right) \rightarrow H_{4}\left(Y^{\prime}\right) \rightarrow H_{2}(X) .
$$

It is known that $Y^{\prime}-Z$ has the homotopy type of a bouquet of 4-spheres and so $H_{4}\left(Y^{\prime}-Z\right)$ is torsion free. It is also known that $H_{2}(X)$ is infinite cyclic (hence torsion free). It follows that $H_{4}\left(Y^{\prime}\right)$ is torsion free. So $I_{0}$ is primitive.

We find similarly for $p_{i}(i=1,2)$ a primitive isotropic rank two sublattice $I_{i}$ in $H_{4}\left(Y_{t}\right)$. We have $I_{i} \perp I_{0}$ for obvious geometric reasons. But $I_{0}^{\perp} / I_{0}$ is positive definite and hence $I_{i}=I_{0}$. We now write $I$ for $I_{0}$.

The image of $H_{4}(L) \cong H_{4}\left(\partial F_{t}\right) \rightarrow H_{4}\left(F_{t}\right)$ is the kernel of the intersection pairing on $H_{4}\left(F_{t}\right)$ and the residual lattice (denoted $\left.Q_{i}\right)$ is of type $E_{6}$. This is also true at the other singular points, so that we have in fact obtained an embedding of $Q:=Q_{0} \perp Q_{1} \perp Q_{2}$ in 
$I^{\perp} / I$. We found in Corollary 2.5 that the equivalence class of a primitive isotropic rank two lattices $J$ in $\Lambda_{o}$ is characterized by the system of (long) roots in $J^{\perp} / J$ and that the root lattice $3 E_{6}$ occurs in this manner. Since there is no root lattice of rank $\leq 18$ that strictly contains $3 E_{6}$, this identifies the equivalence class of $I$.

It remains to see that $Q$ is of index 3 in $I^{\perp} / I$. The discriminant of $E_{6}$ is 3 and hence the one of $Q$ equal to $3^{3}$. Since the square of $\left[I^{\perp} / I: Q\right]$ must divide the discriminant of $Q$, either $Q=I^{\perp} / I$ or $\left[I^{\perp} / I: Q\right]=3$. Let us exclude the former. For this we go back to the decomposed set $\mathcal{B}=\mathcal{B}^{\prime} \sqcup \mathcal{B}^{\prime \prime}$ and the Dynkin diagram $D\left(B_{\ell}\right)$ of long roots it defines. We think of $\mathcal{B}$ as the set of vertices of $B_{\ell}$ of degree 3. We have an injection $r: B_{\ell} \rightarrow \Lambda_{1}$, where we recall that $\Lambda_{1}=e^{\perp} / \mathbb{Z} e$, for some primitive isotropic $e \in \Lambda_{o}$. The full subdiagram on $B_{\ell}-\mathcal{B}^{\prime}$ has 3 connected components, each of type $\hat{E}_{6}$. These components have a common nilvector $e^{\prime} \in \Lambda_{1}$ and we may identify $Q$ with the span of the roots indexed by $B_{\ell}-\mathcal{B}^{\prime}$ modulo $\mathbb{Z} e^{\prime}$. If $u, v \in \mathcal{B}^{\prime \prime}$ are distinct, then it is clear that if we fix a $\hat{E}_{6}$-component, then taking the inner product with $r_{u}-r_{v}$ defines a linear form that takes the value 1 on an extremal vertex, -1 on another extremal vertex and is zero on all other vertices. From this it readily follows that $r_{u}-r_{v}$ is perpendicular to $e^{\prime}$, but not contained in any $\hat{E}_{6}$-summand.

Remark 8.2 The last part of this proof showed that we have a canonical identification of the discriminant groups of the three $E_{6}$-summands: the subgroup $\left(I^{\perp} / I\right) / Q$ lies in $Q^{*} / Q=$ $Q_{0}^{*} / Q_{0} \oplus Q_{1}^{*} / Q_{1} \oplus Q_{2}^{*} / Q_{2}$ as a main diagonal, and thus induces natural isomorphisms between these summands. This helps us to describe the quotient of the orthogonal group $\mathrm{O}\left(I^{\perp} / I\right)$ by the Weyl group $W_{Q}$ of $Q$ (which is indeed normal in $\mathrm{O}\left(I^{\perp} / I\right)$ ): as is wellknown the orthogonal group of $Q_{i}$ is $\{ \pm 1\}$ times its Weyl group. Since -1 acts as such on $Q_{i}^{*} / Q_{i}$, not every element of $\{ \pm 1\}^{3}$ appears here: only its main diagonal (which acts as minus the identity in $Q$ ) preserves $I^{\perp} / I$.

It now easily follows that $\mathrm{O}\left(I^{\perp} / I\right) / W_{Q}$ can be identified with the product of $\{ \pm 1\}$ and the permutation group $\left(\cong \mathcal{S}_{3}\right)$ of the three summands.

We shall now assume that $C$ is generic in the sense that it has no exceptional automorphisms.

Lemma 8.3 The stabilizer $\operatorname{PSL}(V)_{Y}$ of $Y$ in $\operatorname{PSL}(V)$ preserves $\mathbb{P}\left(V^{\prime}\right)$ and $\mathbb{P}\left(V^{\prime \prime}\right)$. It is an extension of the stabilizer of $C$ in $\operatorname{PSL}\left(V^{\prime \prime}\right)$ by the stabilizer of $S_{0} S_{1} S_{2}$ in $\operatorname{SL}\left(V^{\prime}\right)$. The former is the semidirect product of an involution of $C$ in a flex point and the group of order 3 translations in $C, H_{1}\left(C, \mu_{3}\right)$; the latter is a semidirect product of the symmetric group on $S_{0}, S_{1}, S_{2}$ (which we shall identify with $\operatorname{Aut}\left(Y_{\mathrm{sg}}\right)$ ) and a 2-torus. The group of connected components $\pi_{0}\left(\operatorname{PSL}(V)_{Y}\right)$ is a direct product $\operatorname{Aut}\left(Y_{\mathrm{sg}}\right) \times \operatorname{PSL}\left(V^{\prime \prime}\right)_{C}$.

Proof Any projective automorphism $g$ of $Y$ preserves its singular set, and hence the plane spanned by that set (which is defined by putting each $T_{i}$ equal to zero). The action on that plane preserves the coordinate triangle defined by $S_{0} S_{1} S_{2}$. The homomorphism $\operatorname{SL}\left(V^{\prime}\right) \subset \operatorname{SL}\left(V^{\prime}\right) \times \operatorname{SL}\left(V^{\prime \prime}\right) \subset \operatorname{SL}(V) \rightarrow \operatorname{PSL}(V)$ is injective and via this embedding we see the stabilizer of $S_{0} S_{1} S_{2}$ in $\operatorname{SL}\left(V^{\prime}\right)$ faithfully act on $Y$. If $g$ acts trivially on this plane, then we can represent $g$ by a transformation $\tilde{g} \in \mathrm{GL}(V)$ such that $\tilde{g}^{*} S_{i}-S_{i}$ and $\tilde{g}^{*} T_{i}$ are linear combinations of $T_{0}, T_{1}, T_{2}$. Since $\tilde{g}$ multiplies $S_{0} S_{1} S_{2}-\Phi\left(T_{0}, T_{1}, T_{2}\right)$ by a scalar, we see that we must have $\tilde{g}^{*} S_{i}=S_{i}$ for all $i$. It then follows that $\tilde{g}^{*} \Phi=\Phi$. The lemma now follows easily.

Choose a marking of $Y_{t}$. This identifies $H_{1}(C) \cong I$ with a primitive isotropic sublattice of $\Lambda_{o}$. This identification equips $I$ with a natural orientation and that makes it determine 
a rational boundary component of the disconnected domain $\mathbb{D}$ (and hence singles out a component of $\mathbb{D}$ as well). It is known from singularity theory that the local monodromy group of $Y$ (in the space of all cubics) is the subgroup of $\Gamma$ generated by the reflections in the (long) roots in $I^{\perp}$. We therefore denote it by $W_{I^{\perp}}$. It is clearly a normal subgroup of $Z_{\Gamma}(I)$, the group of $\gamma \in \Gamma$ that leave $I$ pointwise fixed.

Any projective automorphism $g$ of $Y$ sends a smooth cubic $Y^{\prime}$ near $Y$ to another such $g\left(Y^{\prime}\right)$. A path in the space of smooth cubics near $Y$ identifies $H_{4}\left(g\left(Y^{\prime}\right)\right)$ with $H_{4}\left(Y^{\prime}\right)$. Another path makes the two identifications differ by an element of $W_{I^{\perp}}$. Thus we get a group homomorphism $\operatorname{PGL}(V)_{Y} \rightarrow Z_{\Gamma}(I) / W_{I^{\perp}}$. This clearly factors through $\pi_{0}\left(\operatorname{PGL}(V)_{Y}\right)$.

Lemma 8.4 The resulting homomorphism $\pi_{0}\left(\operatorname{PGL}(V)_{Y}\right) \rightarrow Z_{\Gamma}(I) / W_{I^{\perp}}$ is an isomorphism.

Proof The proof amounts to a careful comparison between $W_{I^{\perp}}$ and $Z_{\Gamma}(I)$. The group $W_{I^{\perp}}$ acts in $I^{\perp} / I$ as the (finite) Weyl group $W_{Q}$ of the roots in $I^{\perp} / I$ (which is isomorphic to $\left.W\left(E_{6}\right)^{3}\right)$. The kernel $H_{Q}$ of $W_{I^{\perp}} \rightarrow W_{Q}$ is a Heisenberg group: the image of $H_{Q}$ in $\operatorname{Aut}\left(I^{\perp}\right)$ is the abelian group $Q \otimes I$ and the central extension is by the infinite cyclic group $\operatorname{det}(I)$ and given by the obvious antisymmetric map $(Q \otimes I) \times(Q \otimes I) \rightarrow I \wedge I$ (see Sect. (1.1) of [9]). We have thus described a filtration

$$
\operatorname{det}(I) \subset H_{Q} \subset W_{I^{\perp}}
$$

with successive quotients $Q \otimes I$ and $W_{Q}$. If we do similarly for $Z_{\Gamma}(I)$, we get

$$
\operatorname{det}(I) \subset H_{I^{\perp} / I} \subset Z_{\Gamma}(I)
$$

with successive quotients $\left(I^{\perp} / I\right) \otimes I$ and $\mathrm{O}\left(I^{\perp} / I\right)$. The quotient $\left(I^{\perp} / I\right) / Q$ is cyclic of order 3 and if we identify this group with $\mu_{3}$, then $\left(I^{\perp} / I\right) \otimes I / Q \otimes I$ gets identified with $\mu_{3} \otimes I \cong H_{1}\left(C, \mu_{3}\right)$. This is accounted for by the translations of order 3 in $C$ that sit in $\pi_{0}\left(\operatorname{PGL}(V)_{Y}\right)$. According to Remark 8.2, $\mathrm{O}\left(I^{\perp} / I\right) / W_{Q}$ can be identified with the product $\{ \pm 1\} \times \operatorname{Aut}\left(Y_{\mathrm{sg}}\right)$. The first factor is accounted for by the involution in $C$ and the second is in place already. This completes the proof.

Proof of Proposition 7.2 The GL(V)-orbit of $F$ is determined by the $j$-invariant of $C$. The union $\mathcal{O} \subset \operatorname{Sym}^{3} V^{*}$ of such orbits (so with varying $\Phi$ ) is of codimension 21 and an affinelinear section to $\mathcal{O}$ at $F$ is obtained as follows: the ideal $J(\Phi)$ in $\mathbb{C}\left[T_{0}, T_{1}, T_{2}\right]$ spanned by the partial derivatives of $\Phi$ is of codimension 8 and the jacobian algebra $\mathbb{C}\left[T_{0}, T_{1}, T_{2}\right] / J(\Phi)$ is graded with summands in degree $0,1,2,3$ of dimension $1,3,3,1$ respectively. The $\operatorname{SL}\left(V^{\prime \prime}\right)$ stabilizer of $\Phi$ is finite and acts on $J(\Phi)$. Let $J_{0} \oplus J_{1} \oplus J_{2} \subset \mathbb{C}\left[T_{0}, T_{1}, T_{2}\right]$ be a graded linear lift of the first three summands (so of dimension 7) that is equivariant with respect to the finite group $\tilde{H}_{3}$ (which stabilizes $\Phi$ ), put $N_{i}:=\sum_{k=1}^{3} S_{i}^{k} J_{3-k}$ and $N:=\sum_{i=0}^{2} N_{i}$. Then $F+N$ is a linear section to $\mathcal{O}$ that is invariant under the group $\operatorname{Aut}\left(Y_{\text {sg }}\right) \times \tilde{H}_{3}$.

The summand $N_{1}+N_{2}$ describes deformations of $F_{\phi}$ that do not affect the analytic type of the singularity in $p_{0}$ : the local equation in $s_{1} s_{2}+\Phi\left(t_{0}, t_{1}, t_{2}\right)$ is altered by a homogeneous polynomial of degree 3 that lies in $\sum_{i=1}^{2} \sum_{k=1}^{3} s_{i}^{k} J_{3-k}$ (where we view $J_{3-k}$ as a subspace of $\left.\mathbb{C}\left[t_{0}, t_{1}, t_{2}\right]\right)$. It is well-known from singularity theory (splitting of squares) that such deformations do not change the analytic type. On the other hand, the summand $N_{0}$ yields almost the full semi-universal deformation of the simple elliptic singularity $p_{0}$ : we deform in $J_{0} \oplus J_{1} \oplus J_{2} \subset \mathbb{C}\left[t_{0}, t_{1}, t_{2}\right]$ and thus get a codimension one subspace transversal to the 
equisingularity stratum. We shall identify $N_{0}$ with this deformation space. We do likewise for the other cases.

At this point we need to recall our work on the deformation theory of the simple elliptic singularities [8]. Let $G=G_{0}+G_{1}+G_{2} \in N$ with $G_{i} \in N_{i}$ be such that the cubic $Y_{G}$ defined by $F+G=0$ is nonsingular. For simplicity, we assume that each $G_{i}$ is close to zero. Then $p_{i}$ has a Milnor fiber $M_{i} \subset Y_{F+G}$. These Milnor fibers are pairwise disjoint. We have $H_{4}\left(M_{i}\right)$ that is free of rank 8 . The intersection form on $H_{4}\left(M_{i}\right)$ has a rank 2 kernel and the residual lattice is isomorphic to the root lattice of type $E_{6}$. If we merely know that each $G_{i}$ is nonzero, then the singular points of $Y_{G}$ are all of type $A, D$ or $E$ and lie in the (possibly singular) Milnor fiber $M_{i}$. In [8] we considered the period mapping that essentially assigns to $G=G_{0}+G_{1}+G_{2}$ with $G_{i} \neq 0$ for all $i$ the periods of $\omega_{F+G}$ on $H_{4}\left(M_{i}\right)$. We proved there a rather precise Torelli type of result, which may be stated as follows: if $\mathbb{D}(I) \subset \mathbb{D}^{b}$ denotes the rational boundary component defined by the oriented plane $I$, and $U$ is a small transversal slice to $F$ in $\mathcal{O}$, then the period map defines a map

$$
U \times N \rightarrow W_{I^{\perp}} \backslash \operatorname{Star}\left(S, \mathbb{D}^{b b}\right)
$$

that is a local isomorphism along $U$. The map $W_{I^{\perp}} \backslash \operatorname{Star}\left(S, \mathbb{D}^{b b}\right) \rightarrow \Gamma \backslash \mathbb{D}^{b b}=X$ factors through $Z_{\Gamma}(I) \backslash \operatorname{Star}\left(S, \mathbb{D}^{b b}\right)$ and the Baily-Borel construction shows that near a generic point of $\mathbb{D}(I)$, the map $Z_{\Gamma}(I) \backslash \operatorname{Star}\left(S, \mathbb{D}^{b b}\right) \rightarrow X$ is a local isomorphism. On the other hand, the composite map

$$
N \rightarrow W_{I^{\perp}} \backslash \operatorname{Star}\left(S, \mathbb{D}^{b b}\right) \rightarrow Z_{\Gamma}(I) \backslash \operatorname{Star}\left(S, \mathbb{D}^{b b}\right)
$$

has, according to Lemma 8.4, the property that any fiber is contained in a PGL $(V)_{Y}$-orbit and hence in a PGL $(V)_{Y}$-orbit. Similarly, we have that for any other $u \in U$, a fiber of $\{u\} \times N \rightarrow$ $W_{I^{\perp}} \backslash \operatorname{Star}\left(S, \mathbb{D}^{b b}\right) \rightarrow Z_{\Gamma}(I) \backslash \operatorname{Star}\left(S, \mathbb{D}^{b b}\right)$ is contained in a PGL $(V)$-orbit. It follows that the period map separates the orbits near $Y$.

Acknowledgements I thank Bert van Geemen for pointing out to me that my implementation of Vinberg's algorithm for the hyperbolic lattice that is relevant here (and which I use) had already been carried out by its inventor in [12]. I am also indebted to Duco van Straten for supplying me with the classical name of (and a references for) the Perazzo primal. (Incidentally, we establish a connection between the two, for we find that the hyperbolic lattice in question is associated to the Perazzo primal.)

Open Access This article is distributed under the terms of the Creative Commons Attribution Noncommercial License which permits any noncommercial use, distribution, and reproduction in any medium, provided the original author(s) and source are credited.

\section{References}

1. Allcock, D.: The moduli space of cubic threefolds. J. Algebr. Geom. 12, 201-223 (2003)

2. Beauville, A., Donagi, R.: La variété des droites d'une hypersurface cubique de dimension 4. C.R. Acad. Sci. Paris Sér. I Math. 301, 703-706 (1985)

3. Griffiths, Ph.: On the periods of certain rational integrals. I, II. Ann. Math. (2) 90, 460-495 (1969); ibid. (2) 90, 496-541 (1969)

4. Hassett, B.: Special cubic fourfolds. Compos. Math. 120, 1-23 (2000)

5. Huybrechts, D.: Compact hyperkähler manifolds: basic results. Invent. Math. 152, 209-212 (2003)

6. Laza, R.: The moduli space of cubic fourfolds. J. Algebr. Geom. (to appear). Available at arXiv:0704.3256v1[math.AG]

7. Laza, R.: The moduli space of cubic fourfolds via the period map. Ann. Math. (to appear). Available at arXiv:0705.0949v1[math.AG] 
8. Looijenga, E.: On the semi-universal deformation of a simple elliptic singularity I, II. Topology 16, 257-262 (1977); ibid. 17, 23-40 (1978)

9. Looijenga, E.: Compactifications defined by arrangements. II. Duke Math. J. 119, 527-588 (2003)

10. Looijenga, E., Swierstra, R.: On period maps that are open embeddings. J. Reine Angew. Math. 617, 169-192 (2008). Available at math.AG/0512489

11. Scattone, F.: On the compactification of moduli spaces for algebraic $K 3$ surfaces. Mem. Am. Math. Soc. 70(374), (1987), x+86 pp.

12. Vinberg, E.B.: The two most algebraic K3 surfaces. Math. Ann. 265, 1-21 (1985)

13. Voisin, C.: Théorème de Torelli pour les cubiques de $\mathbb{P}^{5}$. Invent. Math. 86, 577-601 (1986). Erratum in Invent. Math. 172, 455-458 (2008)

14. Yokoyama, M.: Stability of cubic hypersurfaces of dimension 3 and 4. Preprint, Nagoya University 